\title{
Study on SDN Technology Based on OpenFlow and Its Application Prospect
}

\author{
Yanhong Chen ${ }^{1, a}$, Peng Zhang ${ }^{1}$, and Shuhua Zhou ${ }^{1}$ \\ ${ }^{1}$ High-tech Research Institute, No.12 Fangongting South Street, Qingzhou City, Shandong Province, China
}

\begin{abstract}
Openflow-based SDN separates the control plane of the network from the data plane of the switch and router, providing a new idea for the future development of the Internet. This paper starts with the connotation of OpenFlow and SDN, analyzes the key technologies of SDN, and prospects the application prospect of SDN.
\end{abstract}

\section{Introduction}

The traditional Internet has become an indispensable part of people's work, study and life after more than 40 years of development. Compared with its flexible and colorful application layer, its network layer is increasingly rigid and closed. The Internet based on TCP/IP couples control logic and data forwarding tightly with the network devices, in most cases it is necessary to configure the router or switch separately. On the contrary surface, some emerging businesses such as the data center, cloud computing, and some new technologies need to join and update kinds of agreements onto the control plane. On one hand the deployment process is complicated and huge, on the other hand it will take a lot of time and energy. In 2006, Nick McKeown, a professor at Stanford university, was inspired by his research project Ethane and proposed the concept of Open Flow, which was a brand-new network architecture concept with Open and programmable as the core. This concept was further extended to Software Defined Networking in 2009. In 2011, the establishment of the Open Network Foundation became an important milestone in the development of SDN/OpenFlow. In 2012, the positive actions of the industry pushed SDN to a climax. The proposal of SDN architecture provides more possibilities for the design, management and use of network resources, and facilitates the innovation and development of network.

\section{Connotation of SDN technology based on OpenFlow}

The Ethane project attempted to use a centralized controller to enable network administrators to easily define network-based security control policies and apply these to various network devices, thus realizing the security control of the entire network communication. Therefore, the earliest concept of OpenFlow is to separate the two functional modules of data plane and control plane of traditional network devices and manage and configure various network devices through centralized controller and standardized interface. Based on the programmable characteristics brought by OpenFlow to the network, professor McKeown further proposed the concept of SDN. The core idea of SDN is to separate the control plane of the network from the data forwarding plane and realize programmable control. The tightly coupled traditional network is divided into three layers: application layer, control layer and infrastructure layer. Control functions are transferred to the server, and the upper application is abstracted into multiple logical entities. By decoupling, the control layer and the forwarding layer are separated and opened, so that the third-party value-added applications can control the network business more intelligently, thus realizing the network programming. A SDN is mainly composed of two devices: controller and OpenFlow switch. The controller allocates a forwarding path for each flow in SDN by collecting statistical data of each OpenFlow switch; The OpenFlow switch sends the flow to the corresponding port according to the instructions of the controller, as shown in figure 1.

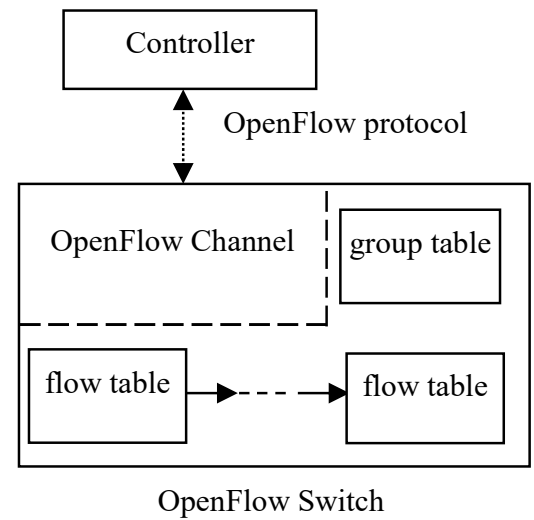

Figure 1. Overall Architecture of OpenFlow

$\bar{a}$ Corresponding author: 395179801@qq.com 
Therefore, the generation of SDN is closely related to OpenFlow protocol. Although strictly defined, OpenFlow only refers to one of a variety of communication protocols between SDN control plane and data plane, in fact, OpenFlow has taken its good flexibility and normalization as the de facto standard of SDN communication protocol, which is similar to TCP/IP as the communication standard of the Internet. Therefore, SDN based on OpenFlow protocol is generally regarded as a narrow SDN in the industry, and this concept is also the default concept in the industry.

\section{Key technologies of SDN based on OpenFIow}

\subsection{Network operating system}

In the SDN category, the Network operating system (NOS) is also called the Network controller. Currently, the popular NOS include NOX, Beacon, Trema, Maestro and so on. All the intelligent and core functions of the network are reflected in the network controller, which schedules and manages the forwarding strategy of the forwarding surface and supports different businesses running on the network controller through the nonintelligent fast forwarding surface equipment. NOS is like the operating system of OpenFlow network, which manages traffic by operating on the switch. Therefore, the switch also needs to support corresponding management functions.

From the perspective of the whole network, NOS should abstract all kinds of resources in the network and provide an easy-to-use interface for network management. It does not complete the management tasks of the network itself, but realizes specific management tasks through various "applications" running on NOS. Managers and developers can focus on developing these applications without spending time analyzing the underlying details. When traffic passes through the switch, if no corresponding matching table item is found, it is forwarded to the network controller running NOS and a decision mechanism is triggered to determine which application the traffic belongs to. Applications running on NOS use traffic information to build a network view and determine traffic behavior. It is because of NOS that SDN has the ability to establish different logical networks and implement different traffic management strategies for different applications.

\subsection{Abstract modeling of forwarding surface}

One of the key technologies of SDN is the abstract modeling of the forwarding surface, which is similar to the Virtual File System (VFS) subsystem in the Linux operating System. VFS abstracts a common file system, providing a unified data structure definition and operation method for the upper layer, and ADAPTS different file systems for the lower layer, such as ext2, ext3, NFS and so on. For the abstract model of SDN forwarding surface, the ONF standard organization proposed and standardized the OpenFlow protocol, in which the forwarding surface device is abstracted as a forwarding model driven by multi-level flow table.

\subsection{OpenFlow protocol}

OpenFlow protocol is the basis for SDN to realize the separation of control and forwarding. In order to promote the development of SDN and unify OpenFlow standard, ONF was established in March 2011. ONF mainly focuses on promoting SDN based on OpenFlow protocol to become a new network standard. Its main research achievements include defining SDN basic architecture, OpenFlow standard and OpenFlow configuration and management protocol. Since the first release of the OpenFlow specification in October 2009, ONF has released 1.1, 1.2, 1.3, and the latest version of OpenFlow 1.4 in October 2013.

The OpenFlow specification is mainly composed of port, flow table, communication channel and data structure, and its data grouping processing principle is as follows. After the SDN switch receives the data packet, it first looks for matching flow entries on the local flow table. Data groups match from the first flow table and may go through multiple flow tables, called pipelining. The flow entry in the flow table contains three parts, matching: matching the existing flow entry according to the input port of data grouping, header field and the information passed by the previous flow table; Count: count the groups that match successfully; Operations: including output grouping to port, encapsulation to controller, discarding, and so on. If a data group successfully matches a flow entry in the flow table, the count of the flow entry is updated and the operation in the flow entry is performed. If not, the first message or message digest of the data stream is forwarded to the controller, which decides the forwarding port.

\subsection{Network virtualization}

Network virtualization enables the creation of logically independent networks on the same Shared physical network resources to meet the application trends of multitenancy, traffic isolation and logical network freedom control. FlowVisor is a network virtualization platform based on OpenFlow controller. By adding virtual abstraction layer to the controller, SDN application layer can only see the local part of the whole network view diagram, provide more abstract network resource description, and realize more flexible programming. From the point of the current research progress, people have used SDN network virtualization advantage in solving cloud computing/data center network overlap, cross subnet IP address of the virtual machine migration, migration across the data center business difficulties, STP/MSTP slow convergence speed, loop link resources waste, unable to multiple tenants provide cross section such as bandwidth issues such as the extensive research and practice.

FlowVisor generates independent network sharding by splitting the flow table space. Network sharding is an 
element of FlowVisor's management function. It is defined by a set of text configuration files. A text configuration file contains rules that control various network activities, ranging from the source IP address of traffic to port number or data packet header information. Through network sharding, FlowVisor divides the physical network into multiple logical networks, allowing multiple controllers to control an OpenFlow switch at the same time, but each controller can control only one virtual network that passes through the OpenFlow switch. Therefore, the test platform established by F1owVisor can allow multiple network tests to be performed simultaneously in different virtual networks without affecting the forwarding speed of the flow. FlowVisor has been widely applied to the experimental platform of multiple research institutions. At the global open network summit, it provides a demonstration environment for various SDN innovative applications to share the same set of physical network resources.

\section{Application prospect of SDN based on OpenFIow}

\subsection{Application of SDN in data center network}

Cloud computing data center is one of the fields with the fastest development of network standards and the most advanced technology types. Especially in the public service cloud, the hosting network which provides users with data isolation by VLAN mode can no longer meet the needs of continuous updating. On the one hand, the number of vlans is limited; On the other hand, users hope to apply for virtual network resources flexibly, including IP address resources, bandwidth resources, firewall, load balancer and other network value-added services resources, just like leasing virtual machines, storage and other resources. Therefore, the new cloud computing data center network should have four capabilities: it can provide a number of scalable isolation networks, and it can provide reusable IP address resources for different tenants; A virtual network with guaranteed bandwidth for different tenants; Provides flexible firewall and load balancing modules for different tenants. SDN architecture can better achieve the above capabilities, data center is undoubtedly the first stage of SDN commercial focus.

\subsection{Application of SDN in optical transmission network}

The most prominent advantage of introducing SDN into optical transmission network is that the SDN controller has a global view of the whole network, can grasp the topology and network state and other information of the whole network, improve the convergence speed and network performance such as delay, and ensure the predictability of system routing and performance. At present, the OpenFlow protocol has been extended to the field of transmission network, adding granular support for time slot, port, label, wavelength and so on. Power, attenuation, nonlinear cost, dispersion cost, and other additional costs related to network physical layer parameters can be abstracted as digital model input SDN controller, which realizes the management and control of network parameters. SDN controllers that master the parameters of the physical layer of the whole network can also select appropriate modulation formats and spectral resources according to the length of optical transmission channel and the number of cross-sections. In addition, there are various types of optical network equipment veneer. If programmable software under SDN architecture and customized speed/code type optical modules are adopted, normalization of veneer hardware can be realized, the number of spare parts can be reduced, and rapid opening and deployment of various types of businesses can be realized.

\subsection{Application of SDN in government and enterprise networks}

Government and enterprise networks have many types of business, complex network equipment functions and many types, which require high security of the network, centralized management and control, high flexibility of the network, and the ability to meet the requirements of customization. The architecture of SDN forwarding and control separation can make network equipment universal and simple. SDN separates the complex business functions and is implemented by the upper application server, which can not only reduce the cost of equipment hardware, but also simplify the enterprise network and make the hierarchy clearer. At the same time, the logic centralization of SDN control can realize the centralized management and control of enterprise network, centralized deployment and management of enterprise security policies, and more flexible customized network functions can be applied in the controller or upper layer to better meet the needs of enterprise network. As the enterprise network is generally constructed, managed and maintained by the enterprise's own information department, which is closed and can be uniformly planned, deployed and upgraded, SDN deployment is highly feasible.

\subsection{Application of SDN in Solid-shift Fusion}

Under the background of full-service operation, the current communication operators have two networks, namely fixed network and mobile network, and the edge gateway of these two networks is assumed by different devices. If SDN technology is applied, the control plane of gateway device can be extracted and uniformly deployed to NOS of cloud data center that also adopts SDN technology. Since the control plane can be programmed by script and the operator can develop it to meet the functional requirements of customers, most of the business can be completed in the control plane. At the same time, the gateway device becomes the forwarding surface device and can act as $x G W / x G X N / B R A S$ simultaneously. Based on the above operations, the data forwarding surface of fixed network and mobile device can be unified, so as to achieve the integration of fixed 
network and mobile network.

\section{Conclusion}

At present, SDN related technologies are not mature enough and the degree of standardization is not high enough. SDN still has a long way to go and network reform must go through a long process. And because the whole industry is facing reshuffle, it is difficult to judge whether each manufacturer can support open standards, and the development of system technology is uncertain. But as a disruptive technology of network, SDN has become a new growth point of IT and network industry. New network industry chain is forming, which will bring about great influence on network hardware and software and network architecture. The technology roadmap that used to be closed to the Internet will certainly change in the future, and the architecture of the network system will become more flexible to support increasingly rich web applications. Network equipment providers, network operators and Internet service providers are paying close attention to the wave of technological revolution brought by SDN. SDN will be an important component and core representative of the network in the future.

\section{References}

1. Xiaojin Tang. Application of SDN and NFV in $5 G$ Mobile Communication Network Architecture (Mobile Information, Issue no.4, 2018)

2. Chaokun Zhang, Yong Cui, Heyi Tang, Jianping Wu. Research Progress of Software Defined Networking (Journal of Software, Issue no.1, 2015)

3. Shunmiao Zhang, Fumin Zou. Research Review on Software Defined Networking (Computer Application Research, August 2013) 